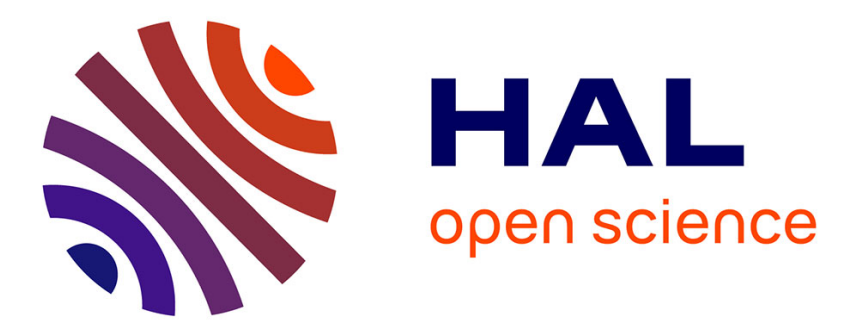

\title{
Bifurcation of magnetic island saturation controlled by plasma viscosity
}

Patrick Maget, Olivier Février, Hinrich Lütjens, Jean-Francois Luciani, Xavier Garbet

\section{- To cite this version:}

Patrick Maget, Olivier Février, Hinrich Lütjens, Jean-Francois Luciani, Xavier Garbet. Bifurcation of magnetic island saturation controlled by plasma viscosity. Plasma Physics and Controlled Fusion, 2016, 58 (5), 10.1088/0741-3335/58/5/055003 . hal-01292516

\section{HAL Id: hal-01292516 https://hal.science/hal-01292516}

Submitted on 23 Mar 2016

HAL is a multi-disciplinary open access archive for the deposit and dissemination of scientific research documents, whether they are published or not. The documents may come from teaching and research institutions in France or abroad, or from public or private research centers.
L'archive ouverte pluridisciplinaire HAL, est destinée au dépôt et à la diffusion de documents scientifiques de niveau recherche, publiés ou non, émanant des établissements d'enseignement et de recherche français ou étrangers, des laboratoires publics ou privés. 


\title{
Bifurcation of magnetic island saturation controlled by plasma viscosity
}

\author{
Patrick Maget, Olivier Février, Hinrich Lütjens ${ }^{1}$, Jean-Francois \\ Luciani $^{1}$, Xavier Garbet \\ CEA, IRFM, F-13108 Saint Paul-lez-Durance, France. \\ ${ }^{1}$ Centre de Physique Théorique, Ecole Polytechnique, CNRS, France. \\ E-mail: custserv@iop.org
}

\begin{abstract}
Two nonlinear regimes, depending on the magnetic Prandtl number Prm, are identified for magnetic islands described by resistive MHD equations. The frontier between these two regimes is sharp, and has the characteristics of a phase transition controlled by plasma viscosity. In the low Prm regime, a new form of the so-called flip instability, consisting of a sudden change of the island phase, is identified. Already known in the context of a forcing by external magnetic perturbations and localized current drive, it occurs spontaneously at low Prm. The main characteristics of this new structural instability are described. The low Prm regime is well described by the slab visco-resistive model in the linear phase, and is characterized by both a large saturation of the island and strong nonlinearly driven zonal flows (that do not impact significantly the island dynamics however), while curvature physics strongly impacts the viscous regime.
\end{abstract}

Published in Plasma Physics and Controlled Fusion 58(5), 055003 (2016) http://stacks.iop.org/0741-3335/58/i=5/a=055003

\section{Introduction}

The saturation of magnetic islands in tokamak plasmas causes a confinement degradation that increases with the island size, so that the prediction of the nonlinear regime is a key issue in fusion research. Although high performance regimes can only be modelled by taking into account the destabilizing contribution of the bootstrap current [1], it is of interest to consider the nonlinear behavior of magnetic islands in the pure resistive MHD framework, as a basis for the understanding of more complex models. A key point is the role of dissipative effects in the saturation. Indeed, simulations are usually performed at a resistivity that is much larger than the experimental one, so that it is crucial to understand how this affects the saturation prediction. Also, the viscosity of tokamak plasmas is not well known, in particular in the direction perpendicular to the magnetic field, so that the role of a dimensionless parameter like the magnetic Prandtl number (Prm $\equiv \mu_{0} \nu / \eta$ in SI units, with $\nu$ the viscosity and $\eta$ the resistivity) needs to be clarified. For example, the threshold for mode locking due to residual 
Resonant Magnetic Perturbations is an area where the amplitude of plasma viscosity could have important implications $[2,3,4,5]$. While viscosity reduces the linear growth rate of tearing modes [6] and introduces a threshold in the stability parameter $\Delta^{\prime}[7,8]$, numerical investigations in cylindrical geometry using a reduced MHD model showed that the nonlinear regime could consist of an oscillation of the island width at low Prm, while the saturation becomes independent of the viscosity above some value [9]. This oscillating regime at low Prm was also observed in slab geometry [10].

In the present work, we address this issue for a single tearing mode in toroidal geometry, with finite perpendicular and parallel transport, using the XTOR code [11]. We find that, depending on plasma viscosity (or magnetic Prandtl number Prm), the island can follow two distinct nonlinear regimes, separated by a sharp transition. In the low viscosity regime, the island enters into a transitory limit oscillation cycle before growing to saturation, and the saturation width does not depend on Prm. This limit cycle is due to a "flip instability" [12], already known in the litterature in the context of external magnetic perturbations [13] or current drive [14]. Above a critical Prm, the island grows regularly (no flip instability) and its saturation size depends strongly on plasma viscosity.

These results have both similarities and differences with previous studies performed in cylindrical and slab geometries $[9,10]$. We also identify two nonlinear regimes, with oscillations of the island width at low Prm, but we find that the island always escapes from the oscillating regime. We also find that the saturation is large and does not depend on Prm in the low viscosity regime, while it was reported that the saturation was small at low Prm and large, as well as independent of viscosity, at high Prm.

In the present work, we identify the mechanisms that are at play in the two nonlinear regimes and impact both the linear and nonlinear phases. At low viscosity, the tearing mode is qualitatively well described by the slab visco-resistive model [7] because curvature stabilization is not sensitive to plasma viscosity. In the viscous regime, on the contrary, curvature physics plays an essential role, both in the linear and nonlinear phases, because the perturbed pressure is influenced by plasma viscosity through the convection of plasma density.

The paper is organized as follows: in section 2, the resistive MHD model is presented, as well as the magnetic equilibrium. A description of the two nonlinear regimes is given in section 3, both for their specific nonlinear dynamics and for the magnetic island saturation dependence on the magnetic Prandtl number Prm. A detailed description of the spontaneous flip instability is given in section 4, evidencing the phase transition when approaching the viscous regimes. The role of plasma viscosity in the linear and nonlinear properties of the tearing mode, and the implication of magnetic curvature, is explained in section 5 , and a conclusion follows. 


\section{MHD model and magnetic equilibrium}

For the purpose of our study, we use the single fluid model implemented in the nonlinear MHD code XTOR [11]. It solves the following equations:

$\left[\partial_{t}+\mathbf{V} \cdot \nabla\right] \rho=-\rho \nabla \cdot \mathbf{V}-\nabla \cdot \Gamma_{a n}+\Sigma$

$\left[\partial_{t}+\mathbf{V} \cdot \nabla\right] p=-\Gamma p \nabla \cdot \mathbf{V}+(\Gamma-1)[H-\nabla \cdot \mathbf{q}]$

$\rho\left(\partial_{t}+\mathbf{V} \cdot \nabla\right) \mathbf{V}=\mathbf{J} \times \mathbf{B}-\nabla p+\nu \nabla^{2} \mathbf{V}$

$\partial_{t} \mathbf{B} \quad=-\nabla \times \mathbf{E}$

$\mathbf{E}+\mathbf{V} \times \mathbf{B} \quad=\eta\left(\mathbf{J}-\mathbf{J}_{\mathbf{0}}\right)$

with $\mathbf{V}=\mathbf{V}_{\mathbf{E}}+\mathbf{V}_{\| \mathbf{i}}, \mathbf{V}_{\mathbf{E}}=\mathbf{E} \times \mathbf{B} / B^{2}, \nu$ the plasma viscosity, $\eta$ the plasma resistivity and $\mathbf{J}_{\mathbf{0}}$ the equilibrium current density. The ratio of specific heat is $\Gamma=5 / 3, H$ is the heat source and $\mathbf{q}=-\rho \chi_{\|} \nabla_{\|} T-\rho \chi_{\perp} \nabla_{\perp} T$ is the heat flux, with $T=p / \rho$ and $p=p_{e}+p_{i}$. The anomalous particle flux is defined as $\Gamma_{a n}=-D_{\perp} \nabla \rho+\rho \mathbf{V}_{\text {pinch }}$ with $D_{\perp}$ the perpendicular diffusion coefficient, and a prescribed pinch velocity $\mathbf{V}_{\text {pinch }}=D_{\perp} \nabla \rho_{0} / \rho_{0}$ inside $\sqrt{\psi}=0.97$, so that the particle source is cancelled in the plasma core. Here, $\rho_{0}$ is the initial density profile and $\psi$ is the normalized poloidal magnetic flux. This pinch is decreased to zero at the edge $(\sqrt{\psi}>0.97)$, and the particle source term $\Sigma$ then drive the density gradient:

$$
\Sigma=\nabla \cdot\left(-D_{\perp} \nabla \rho_{0}+\rho \mathbf{V}_{\text {pinch }}\right)
$$

We set up a tokamak magnetic equilibrium with circular cross-section and inverse aspect ratio $\epsilon=0.3$, using the equilibrium code CHEASE [15]. The pressure profile is given by $\partial_{\psi} p \propto(1-\psi)$ and the current density profile by $I^{*} \propto(1-\psi)^{2}$ (see [15] for the definition of $I^{*}$ ). The position of $q=2$ is prescribed at $\sqrt{\psi}=0.5$, which corresponds to $x \equiv \sqrt{\Phi} \approx 0.34$ and the magnetic shear there is $s=0.585$. The initial density profile is prescribed in the following analytical form

$$
\rho_{0}(\psi)=\frac{1-d_{1} \psi^{d_{2}}}{1+d_{3} \psi^{d_{4}}}
$$

with $\left(d_{1}, d_{2}, d_{3}, d_{4}\right)=(0.32,1,0.1,4)$. The resulting equilibrium has a monotonic $q$ profile with $q(0)=1.56$, and it is characterized by $\beta_{p}=1.12, \beta_{t}=0.15 \%, \beta_{N}=0.84$ and $l_{i}=1.58$.

\section{The two nonlinear regimes}

The nonlinear dynamics of the unstable equilibrium has been investigated in a wide range of plasma viscosities, with a magnetic Prandtl number Prm $\equiv \nu / \eta \in[0.1,100]$. Diffusivities are chosen so that $\eta / \chi_{\perp}=150, D_{\perp}=(2 / 3) \chi_{\perp}$ and $\chi_{\|} / \chi_{\perp}=10^{8}$.

An overview of the numerical simulations is given in figure 1 , with the evolution of the island size on $q=2$ for a Lundquist number at magnetic axis $S_{0}=10^{7}$. Cases corresponding to the low viscosity regime are on the left plot, while cases corresponding to viscous tearing modes are on the right plot. The figure shows that these nonlinear 


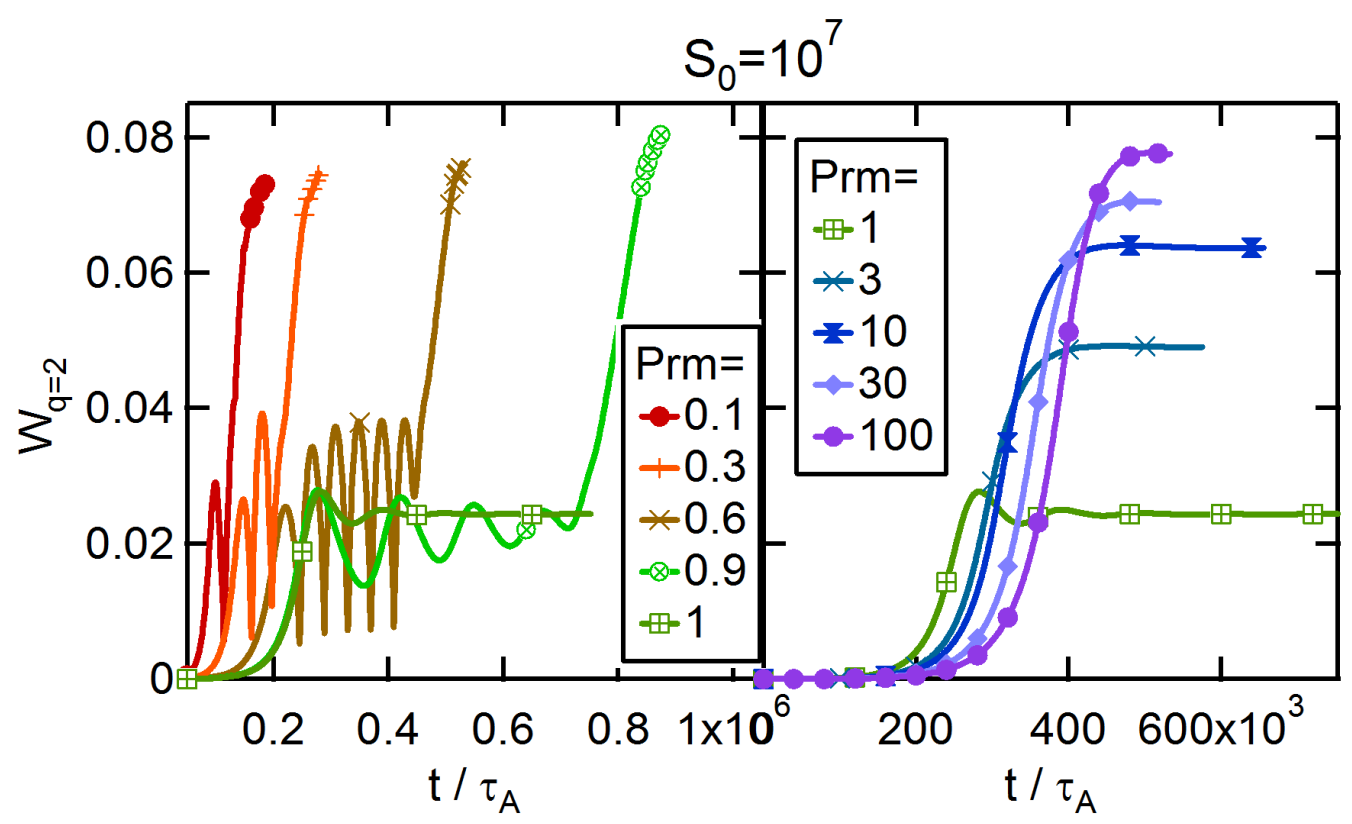

Figure 1. Dynamics of the magnetic island size at $q=2$ for $\operatorname{Prm} \in[0.1,1]$ (left), Prm $\in[1,100]$ (right).

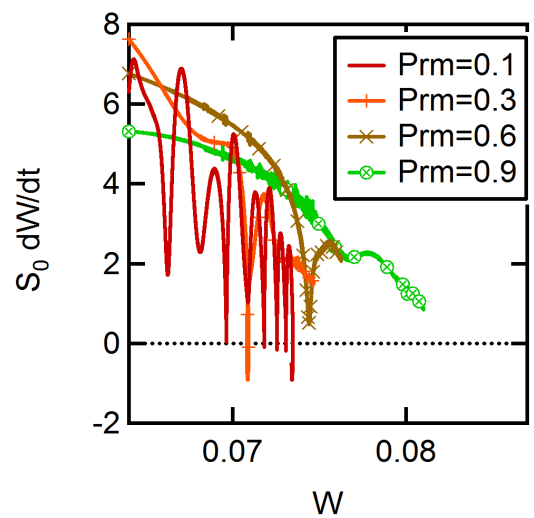

Figure 2. Zoom on the phase diagram $(d W / d t, W)$ close to saturation for Prm $\in$ $[0.1,0.9]\left(S_{0}=10^{7}\right)$.

regimes are extremely different. At low Prm, the saturation size is $W_{\text {sat }} \approx 7-8 \%$, and it is weakly dependent from the magnetic Prandtl number. Note that, at the latest stage of the island dynamics, the progress in the simulations is extremely slow. But although the saturation is not fully completed, the traces of the island decay suggest that it will not increase much above the value that has been reached, in the range $W_{\text {sat }} \approx 0.075-0.08$ (figure 2).

In this low viscosity regime, the island size experiences multiple rebounds before reaching its final saturated state, a phenomenon known as the flip instability (see 

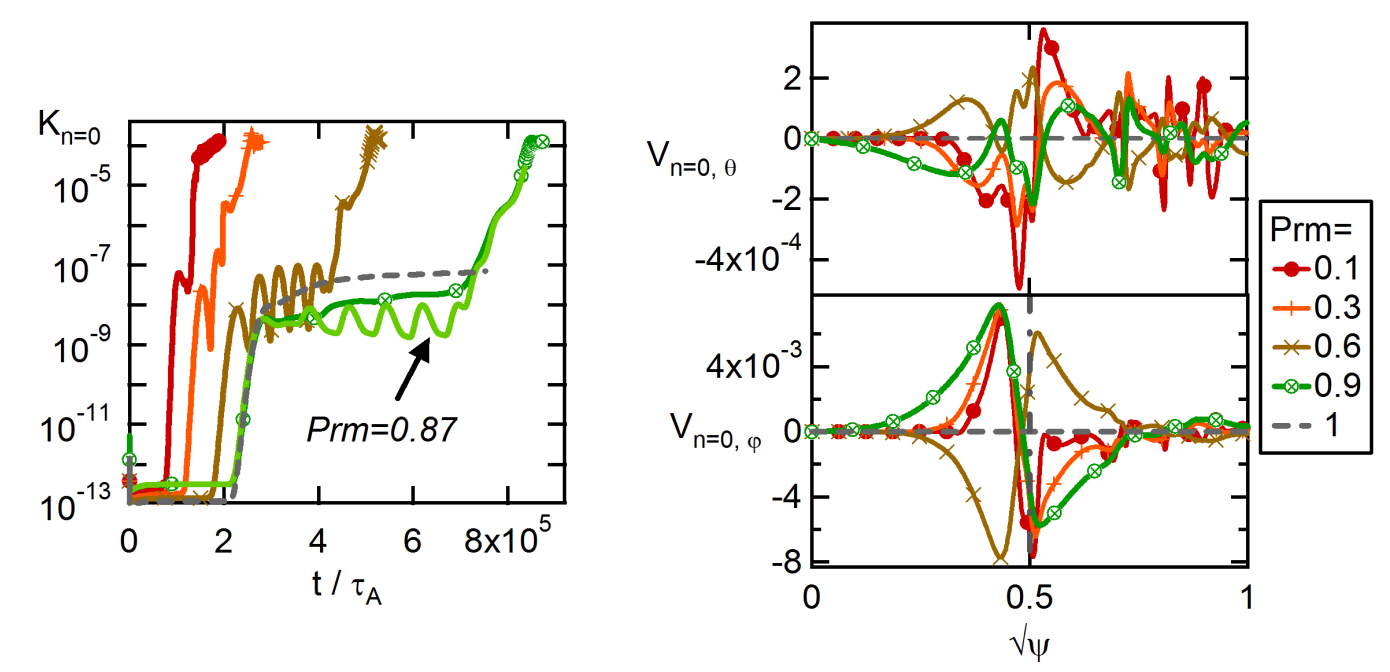

Figure 3. Dynamics of $n=0$ kinetic energies (left); profiles of $n=0$ poloidal and toroidal flows at the end of the simulations (right) for $\operatorname{Prm} \in[0.1,1]$.

section 4$)$. The end of the cycling behaviour coincides with the development of strong $(m=0, n=0)$ (zonal) flows (figure 3). In the toroidal direction, these zonal flows are essentially bipolar relative to the resonant surface $q=2$ located at $\sqrt{\psi}=0.5$, but it can take indifferent directions as shown by the case at $\operatorname{Prm}=0.6$, depending presumably on small differences in the initial noise-level state. The shear of the zonal flows, evaluated in the island region at the end of the simulation, is decreasing with Prm, and vanishes at $\operatorname{Prm}=1$, where the zonal flow generation is insignificant (figure 4). We observe that the slight increase of the saturated island width as this shear decreases is reminiscent of experimental and heuristic indications that flow shear could reduce the saturation of magnetic islands $[16,17]$.

Simulations where the $n=0$ component of the flow is artificially suppressed can answer the question of the role of flow shear in the island dynamics. Due to the long duration of nonlinear simulations close to saturation in this low Prm regime, we only focus here on the dynamics before saturation. This exercice has been done for the case $\operatorname{Prm}=0.1$. We observe that, qualitatively, the characteristics of the nonlinear evolution remains nearly unchanged: the flip instability occurs as before, and the island still exits the flip cycle (figure 5). The generation of zonal flows therefore coincides with the end of the flip cycle without causing it. The only change is a slower initiation of the flip, and a characteristic flip time (as defined later) that is about three times longer: the flow shear clearly acts in the direction of a faster, more unstable dynamics $[18,19]$. Then, the island grows towards a large saturation as before. We can therefore conclude that although nonlinearly driven zonal flows are specific to the low viscosity regime, they do not impact significantly the island dynamics.

An abrupt change occurs above a critical Prm, somewhere in the range Prm $\epsilon$ 


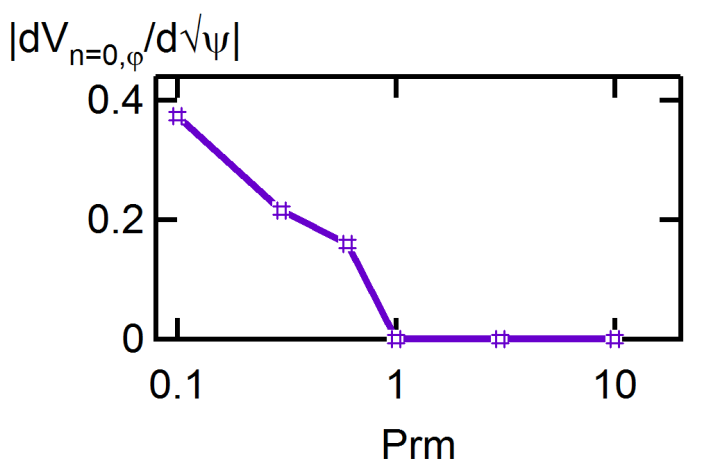

Figure 4. Absolute value of the zonal flow shear in the island region at saturation, as a function of Prm.

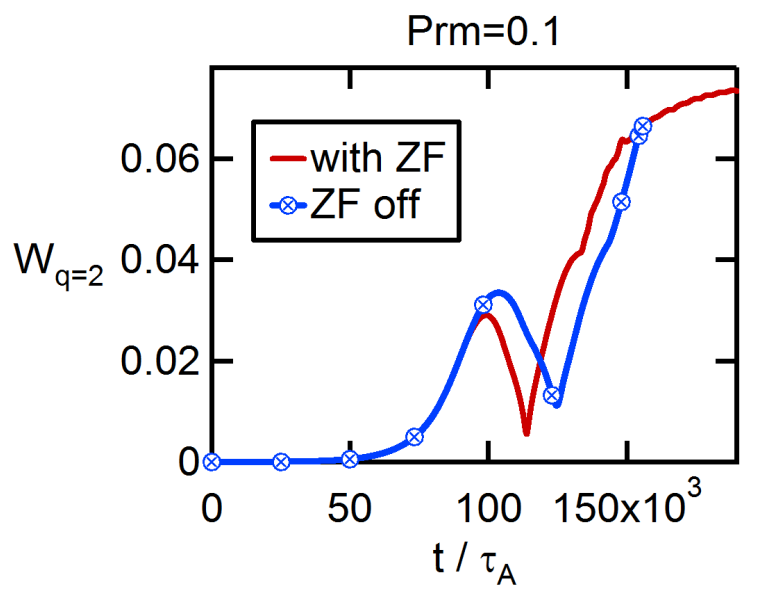

Figure 5. Evolution of the island width with and without zonal flows (ZF), for the case $\operatorname{Prm}=0.1$.

$[0.9,1]$. At Prm $=0.9$, just below the threshold, we observe an intermediate regime where the island slowly oscillates around the low saturation value obtained at $\operatorname{Prm}=1$, without any flip, before rising to the high saturation value of about $8 \%$. Just above the critical value, the island saturates at a much smaller value, around $W_{\text {sat }} \approx 2.4 \%$, and no bouncing of the island size is observed. But the island saturation is no longer independent from viscosity in this viscous regime, and as Prm is further increased, it gradually increases. Note also that the level of zonal flows remains extremely low in this regime.

The dependence of the saturation size of the $(2,1)$ island on Prm is summarized in figure 6 . The saturation can be compared with two important characteristic widths: the visco-resistive width and the curvature width. The visco-resistive width $\delta_{\eta \nu} \propto \operatorname{Prm}^{1 / 6} \eta^{1 / 3}$ [6] is evaluated from the computed fields as the distance between inflexion points of the perturbed poloidal magnetic field, as described in [20]. At $S_{0}=10^{7}$, it is very well fitted by $\delta_{\eta \nu}=0.0172 \mathrm{Prm}^{1 / 6}$, and the saturated island width is always above this 


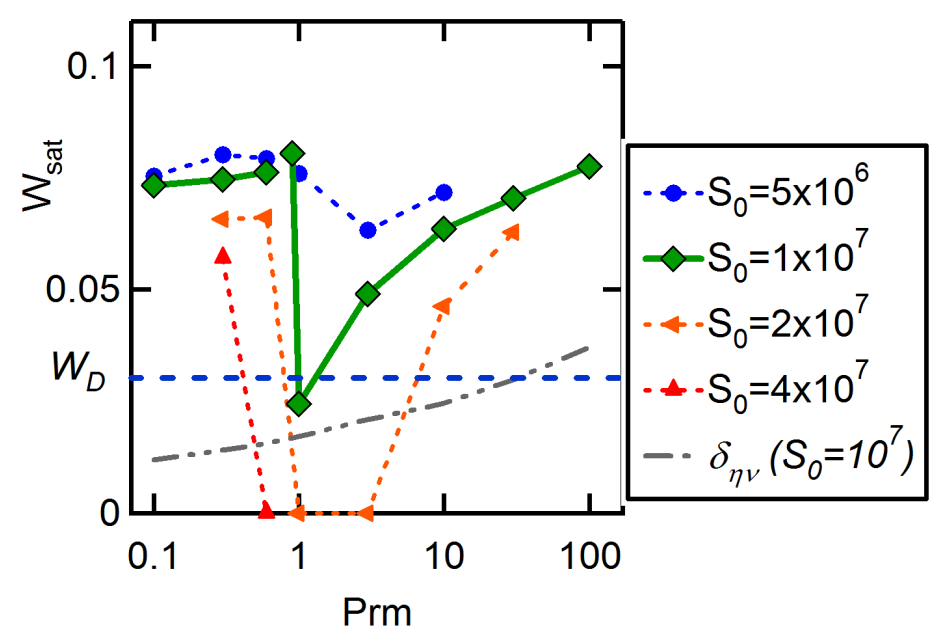

Figure 6. Saturation size as a function of Prm for increasing Lundquist number $S_{0}$. The critical width for curvature $W_{D}$ is shown, as well as the visco-resistive width $\delta_{\eta \nu}$ for $S_{0}=10^{7}$. Note that low Prm cases at $S_{0}>10^{7}$ are not fully saturated.

size. The critical size above which curvature effect decreases is given by $W_{D}=0.81 W_{\chi}$ [21] where $W_{\chi}=2 \sqrt{2}\left(\chi_{\perp} / \chi_{\|}\right)^{1 / 4} \sqrt{x /(\epsilon n s)}, x=\sqrt{\Phi}$ the radial co-ordinate with $\Phi$ the normalized toroidal magnetic flux, $\epsilon=a / R_{0}$ the inverse aspect ratio, $n$ the toroidal mode number, $s=d(\log q) / d(\log x)$ the magnetic shear and $q$ the safety factor. In our case, $W_{D} \approx 0.03$ for the $(2,1)$ mode. Note that the saturation width is mostly larger than the critical size $W_{D}$ above which curvature effect becomes weaker, so that the importance of curvature stabilization $[22,23]$ is expected to be generally moderate. However, viscosity does affect this critical size, as we will see in section 5 , so that in the viscous regime $(\operatorname{Prm}>1)$ curvature stabilization is progressively modified.

\section{The low viscosity regime and the spontaneous flip instability}

One of the particularities of the low viscosity regime is the spontaneous flip instability, and we will show that the transition to the viscous regime is characterized by a singularity in the flip properties, so that the change of regime can be associated with a bifurcation in the nonlinear dynamics of the tearing mode.

\subsection{Phase diagram of the flip instability}

The flip instability can be described as follows: the regular island growth starts at some stage (around $2.5 \%$ of the minor radius here) to turn into decay until it reaches a small size (less than $1 \%$ of the minor radius). Then the decay is moved to a symmetric growth within a very short time (in the range $1000-1500 \tau_{A}$ at $S=10^{7}$ ). This time is evaluated by considering the duration between two maxima of the island growth $d W / d t$ (see figure 


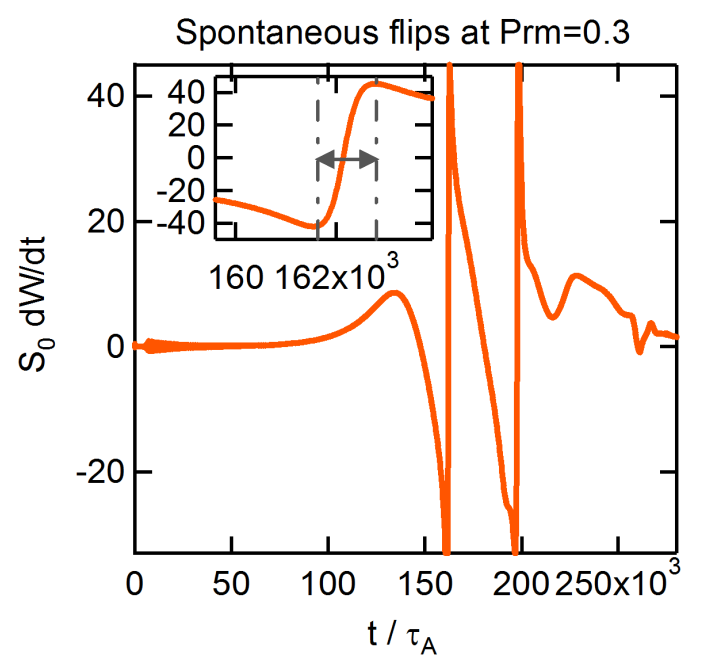

Figure 7. Evolution of the $(2,1)$ island growth $S_{0} d W / d t$ at $\operatorname{Prm}=0.3$, showing the sudden changes corresponding to the spontaneous flips. The zoom shows the flip time scale of about $1000 \tau_{A}$.

7). The flip occurs several times before the island finally leaves this cycle and reaches saturation. But the number of such cycles increases with Prm as the transition to the viscous regime is approached (see figure 1).

The representation of this flip cycle in terms of phase diagram $(d W / d t, W)$ (figure 8) or $(M, K)$ with $M$ the magnetic energy and $K$ the kinetic energy of the $(2,1)$ mode (figure 9 ), suggests that as viscosity is increased to a critical value $\operatorname{Prm}^{\text {crit }} \sim 0.9$, the system has not enough power to enter the limit cycle, a phenomenology that is reminiscent of a pendulum oscillating in a viscous medium. This nonlinear dynamics is exactly similar to that modelled in [13] for the flip induced by an external magnetic perturbation, where it could be modelled by a system of coupled differential equations relating the island width $W$ to its phase $\Phi$ relative to that of the external field. The drive for the spontaneous flip instability has been investigated in cylindrical geometry where it was attributed to the contribution of the inertia term $\rho \mathbf{v} \cdot \nabla \mathbf{v}$ in the momentum equation [9]. Repeating the same test, we find that this term does contribute to increase the number of flips, but the flip instability does not rely on this nonlinearity to exist (figure 10). Other nonlinear terms therefore play a role in this phenomenon, which remains specific to the low viscosity regime.

The characterization of the flip in terms of time scale and period is shown in figure 11 (left plot). The flip time is weakly dependent on Prm, and of the order of $1000 \tau_{A}$, while the flip period progressively increases like $\Delta T^{f l i p} \propto\left|\operatorname{Prm}^{\text {flip }}-\operatorname{Prm}\right|^{-0.3}$ with $\operatorname{Prm}^{\text {flip }} \approx 0.9$. The properties of the flip instability therefore show a bifurcation when approaching the transition to the viscous regime. Note also that the flip time increases with both the central Lundquist number $S_{0}$ and Prm value (figure 11, right). 


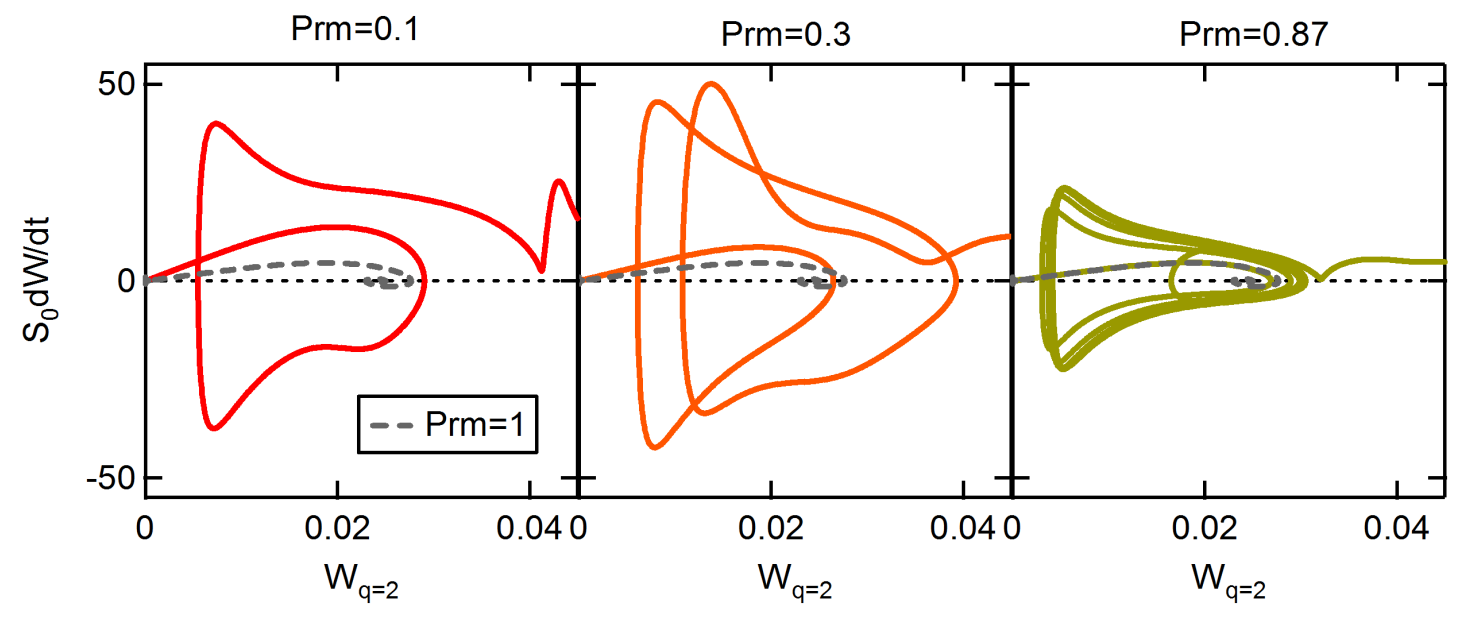

Figure 8. Phase diagram $(W, d W / d t)$ for Prm $=0.1,0.3$ and 0.87 (close to the critical Prm). The case Prm=1 is shown (dotted line) for comparison.

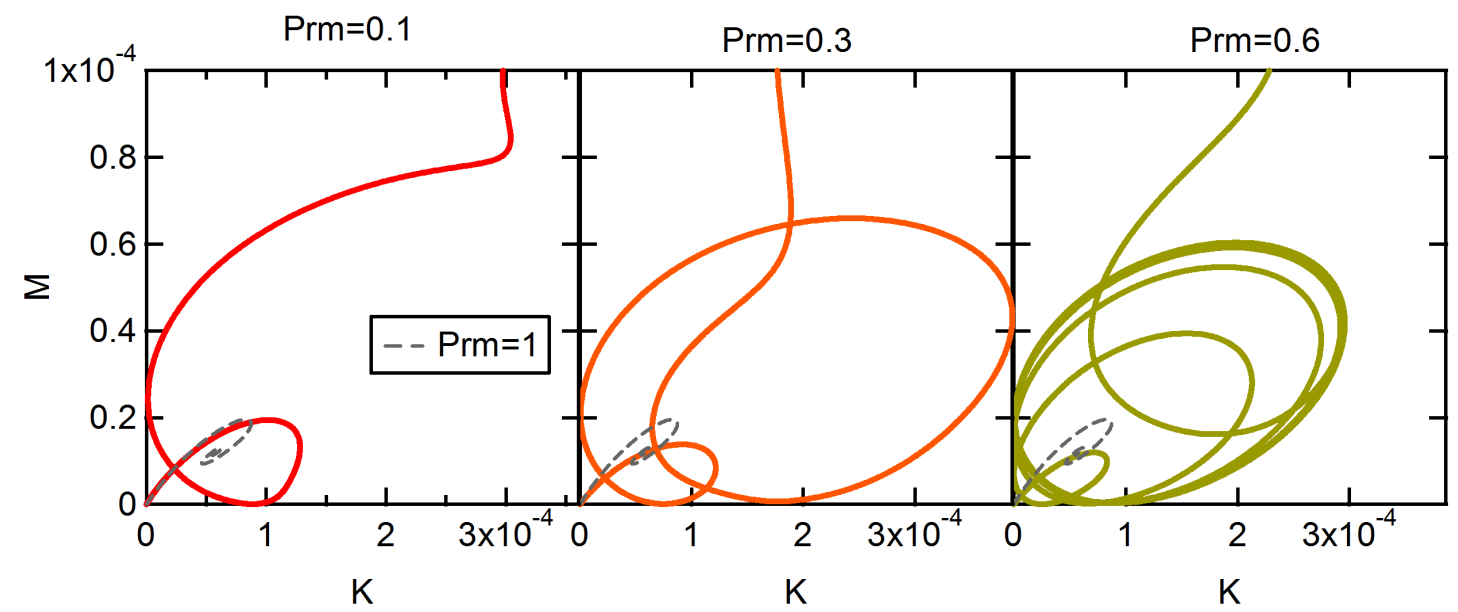

Figure 9. Phase diagram $(M, K)$ with $M$ the magnetic energy and $K$ the kinetic energy of the $(2,1)$ mode, for $\operatorname{Prm}=0.1,0.3$ and 0.6 . The case Prm= 1 is shown (dotted line) for comparison.

\subsection{Reconnection pattern during the flip instability}

The evolution of the island topology during the spontaneous flip is followed by computing Poincaré maps. This is shown in the region of $q=2$ in figure 12 for the second flip of the case $\operatorname{Prm}=0.6$. At the initial X-point positions, small islands form and progressively grow, while the main islands decrease. At the end of the flip, the secondary islands have entirely replaced the initial ones.

The positions of island X-points are recorded at $q=2, q=3, q=4$ and $q=5$ (figure 13). We recognize on $q=2$ the two initial X-points that split around $t=288 \times 10^{3} \tau_{A}$, and the secondary islands that are growing there while the initial ones progressively 


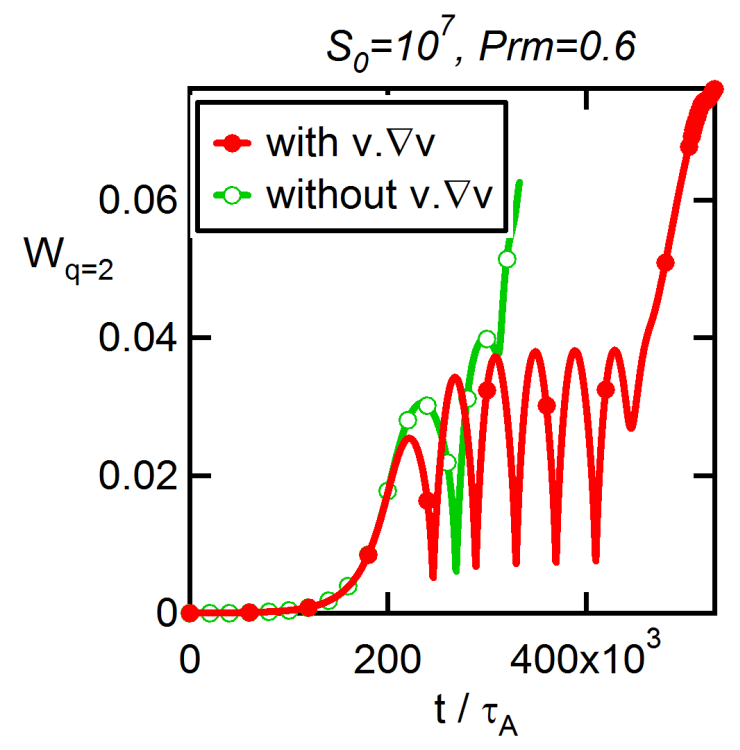

Figure 10. Effet of term $(\rho \mathbf{v} \cdot \nabla \mathbf{v})$ on the spontaneous flip.
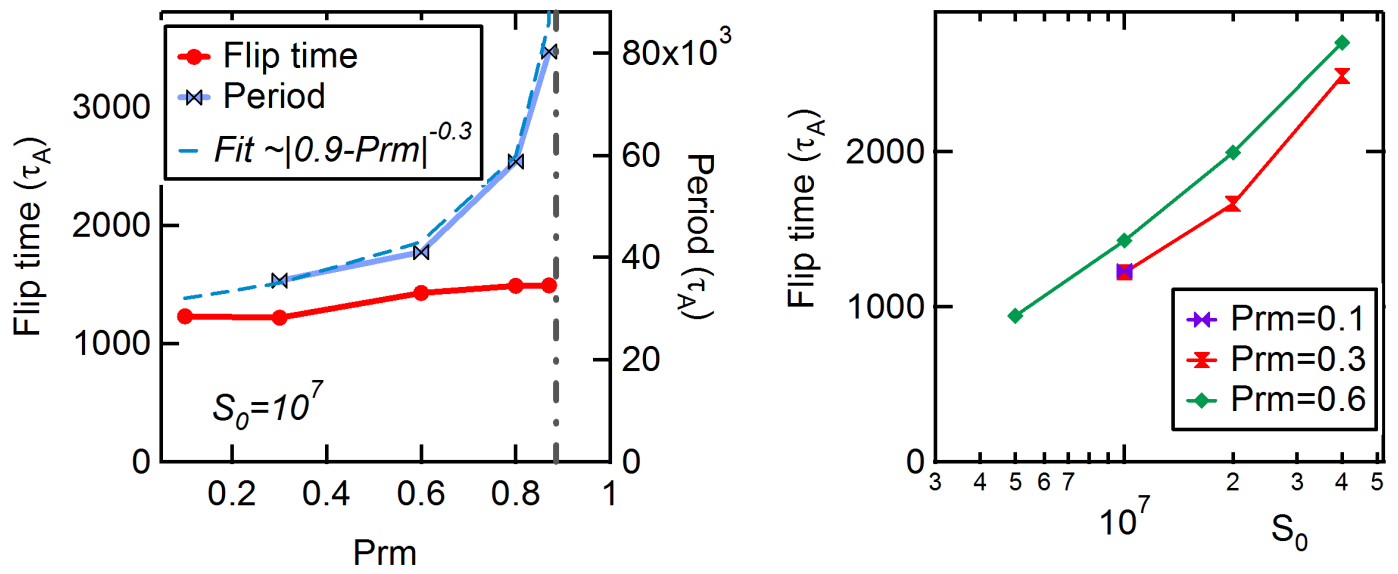

Figure 11. Left: Flip time (left scale) and flip period (right scale) as the critical Prm is approached (vertical dashed line). Right: Time duration of the flip as a function of the central Lundquist number $S_{0}$ for different Prm values.

shrink. After about $3000 \tau_{A}$, around $t \approx 291 \times 10^{3} \tau_{A}$, the new islands have entirely replaced the previous ones. Note that this phenomenon is not equivalent to a rotation of the mode, so that the situation is different from that of an island interacting via viscous forces with a rotating plasma $[24,2]$. This is a structural change, and once it is completed, the X-points do not move.

An interesting feature of the flip at the main unstable resonance, $q=2$, is that it is influenced by other flips occurring at neighbouring resonant surfaces, through the coupling of poloidal harmonics in toroidal geometry. If we consider $q=3, q=4$ and 

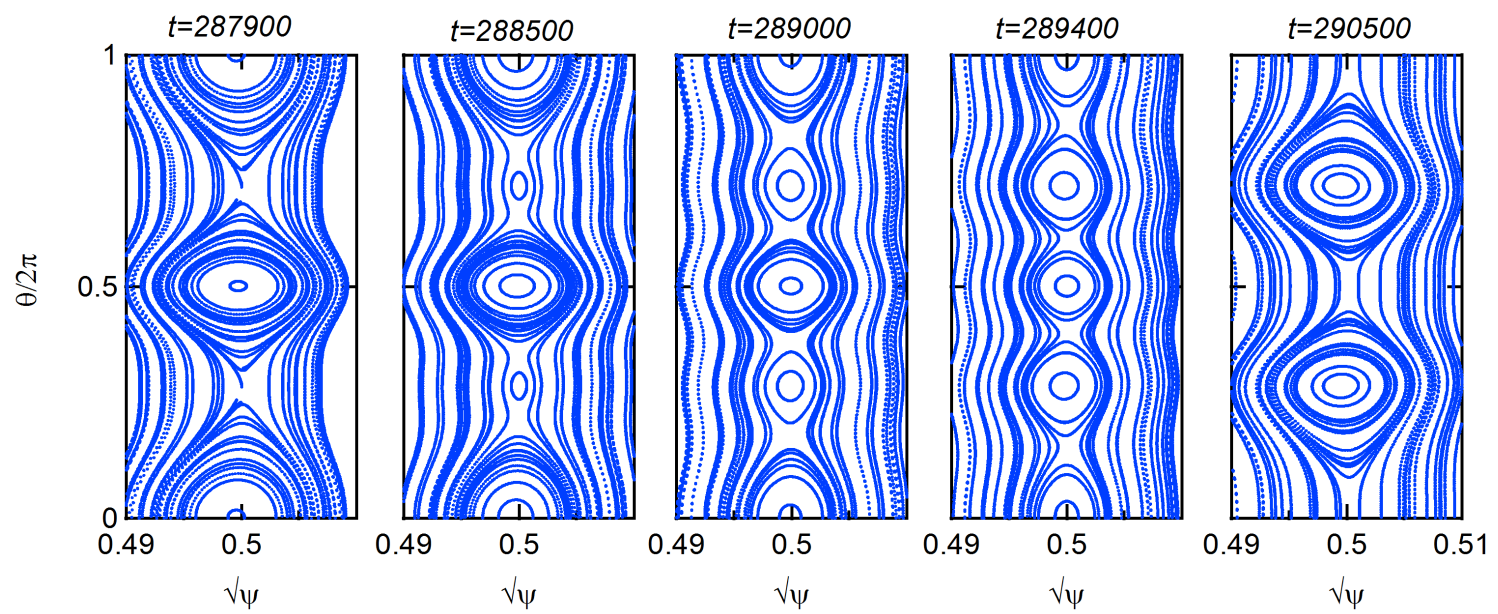

Figure 12. Poincaré maps around $q=2$ during the second spontaneous flip at $\operatorname{Prm}=0.6$.

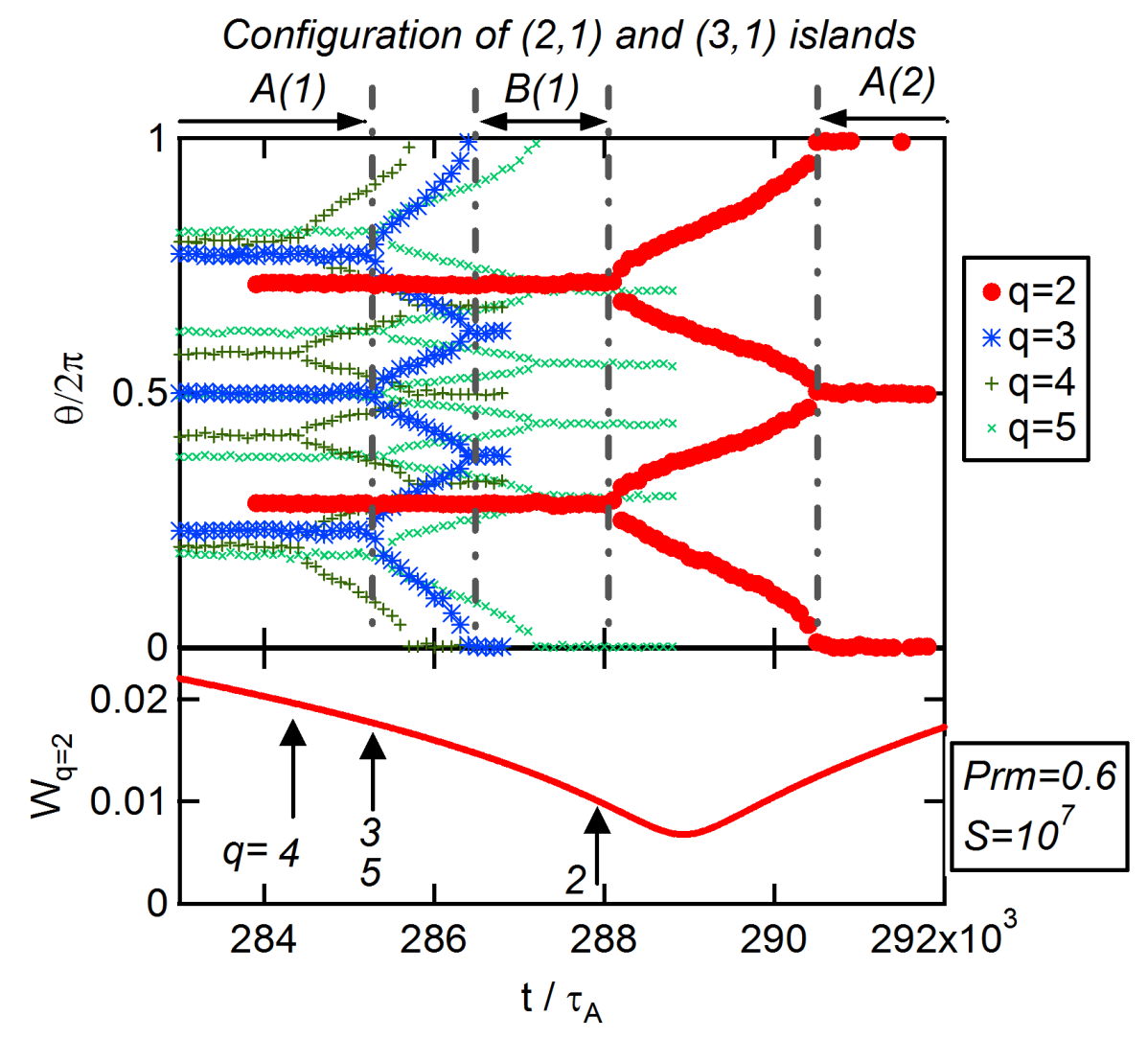

Figure 13. X-point positions at $q=2, q=3, q=4$ and $q=5$ during the flip (top), island size at $q=2$ (determined from the magnetic energy of the $(2,1)$ mode) and onset times (arrows) for flips at $q=2,3,4$ and 5 (bottom). 


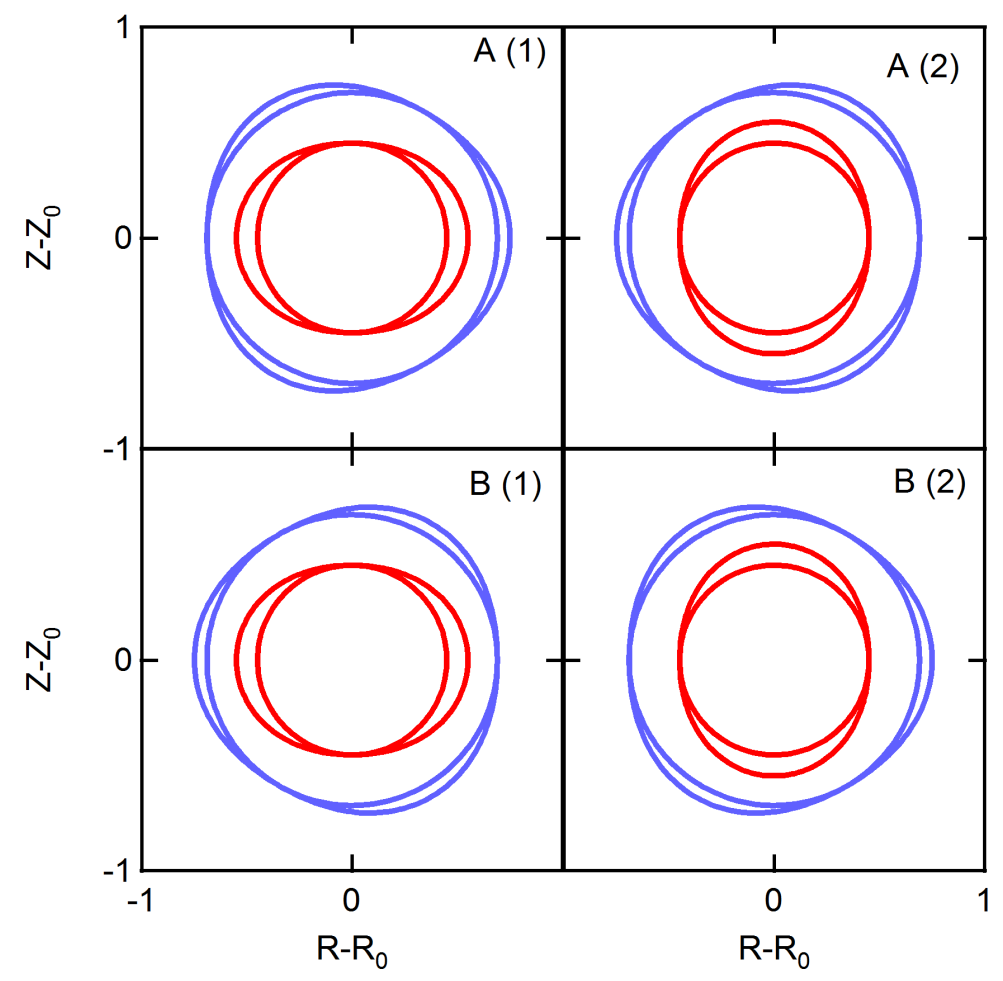

Figure 14. Two possible configurations for $(2,1)$ and $(3,1)$ islands, at $\varphi=0$ (left) and $\varphi=180^{\circ}$ (right).

$q=5$, we notice that the first flip occurs at $q=4$, that its starts at $q=5$ at the same time as at $q=3$, before that of the dominant $(2,1)$ mode. The spontaneous flip instability that we observe on $q=2$ is therefore the last of a series of earlier flips occurring on subdominant poloidal harmonics of the $n=1$ mode to which the $(2,1)$ mode is coupled via toroidal curvature. In fact, the flip on $q=2$ is likely a consequence of the flip occurring at $q=3$. Indeed, the relative position of magnetic islands on both surfaces is not arbitrary. We display in figure 14 the two simplest configurations for the respective positions of $(2,1)$ and $(3,1)$ islands. The configuration noted " $\mathrm{A}$ " is the one that the $n=1$ mode chooses naturally in simulations, probably because it corresponds to a lower energy state. On the temporal evolution of the X-point positions, we observe that the $(3,1)$ island changes its phase before the $(2,1)$ island, so that we move from configuration A-1 at $\varphi=0^{\circ}$ to configuration B-1. The system remains in configuration B-1 during less than $2400 \tau_{A}$, and then the flip of the $(2,1)$ island starts so that the system is back to the original configuration A. The flip on $q=2$ could well be driven so as to minimize the potential energy state of the plasma by returning to its natural configuration with respect to its poloidal sidebands. 


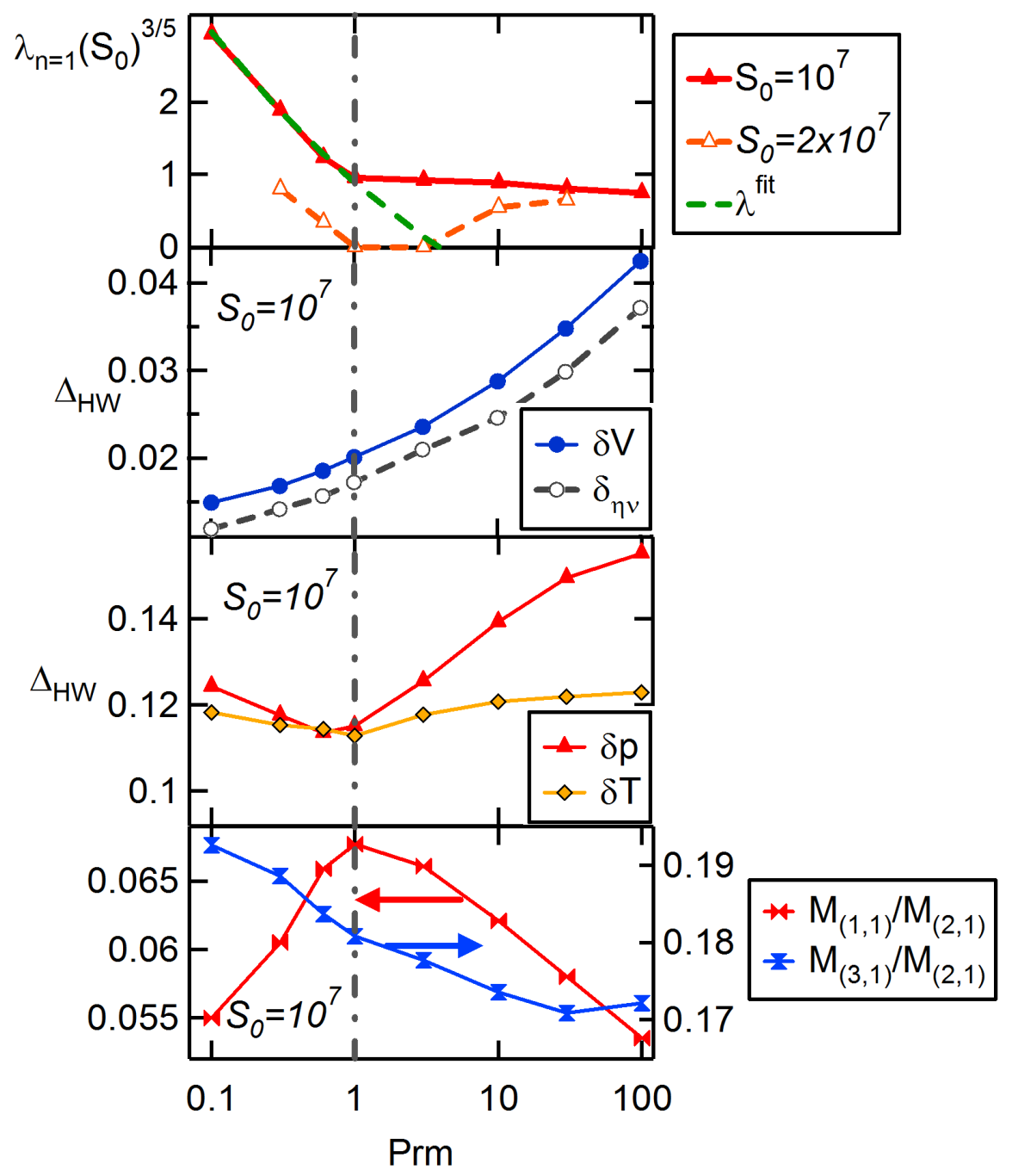

Figure 15. From top to bottom: Linear growth rate of $n=1$ mode and analytical fit following [7], half-width of perturbed radial velocity compared with the measured visco-resistive width $\delta_{\eta \nu}$, half-widths of perturbed pressure and temperature, and contribution of side-band magnetic energies, as a function of the magnetic Prandtl number Prm. The vertical dashed line separates the two saturation regimes.

\section{Relation with linear stability properties and curvature stabilization}

The two nonlinear saturation regimes have in fact strong connection with the linear properties of the tearing mode. We display in the first three plots of figure 15 the linear growth rate of the $n=1$ mode, and the half-width of radial velocity, pressure and temperature perturbations, as a function of the magnetic Prandtl number Prm. These quantities are determined in the linear phase of the mode evolution, prior to any deviation from the exponential growth. 
We first note that the width of the radial velocity perturbation, i.e. of the electrostatic potential, follows that of the visco-resistive width $\delta_{\eta \nu}$ in all the viscosity domain considered. The low viscosity regime is characterized by a growth rate that strongly decays with Prm, and a pressure perturbation that slightly decays. The dependence of the linear growth rate with Prm can be fitted (for $S_{0}=10^{7}$ ) with $\lambda^{f i t} S_{0}^{3 / 5}=4.44\left(\mathrm{Prm}^{-1 / 6}-0.8\right)$, thus yielding a good agreement with the analytical dependencies obtained from slab analytical theory [7]. The tearing mode should become linearly stable for Prm of order unity, in agreement with the transition value observed in our simulations. We note that a transition in the linear properties of the tearing mode has also been found around a magnetic Prandtl number of unity in [25].

The viscous regime, above $\operatorname{Prm}=1$, is therefore of a different nature. The main difference is on the structure of the pressure perturbation, which now increases with Prm. There are two components in the pressure perturbation: one is driven by the temperature part, and the other by the density contribution. Due to the large parallel diffusivity, the structure of the temperature follows that of the magnetic perturbation, and is not sensitive to plasma viscosity, as shown in figure 15. But the density is mainly convected by the flow, and will be affected by the increase of Prm in a similar way as the radial velocity perturbation shown in figure 15 . Thus the change of the pressure perturbation width around $\operatorname{Prm}=1$ means that pressure moves from a regime that is essentially driven by diffusion (low Prm) to a regime where convection takes a larger part. The role of the pressure perturbation in the tearing dynamics comes from curvature physics. In the linear regime, it has a stabilizing influence that is all the more important as the perturbation is localized [26, 23]. In the low viscosity regime, the radial extent of the pressure perturbation does not vary significantly so that curvature physics is expected to be nearly independent of Prm, a situation that explains the good agrement with slab theory that is found. In the viscous regime, the broadening of $\delta p$ weakens curvature stabilization and therefore tends to make the mode more unstable. At $S_{0}=10^{7}$, this trend is balanced by the viscous stabilization, resulting in a growth rate that decays slowly with Prm, but at $S_{0}=2 \times 10^{7}$ and above, the loss of curvature stabilization results in the destabilization of the tearing mode at increasing Prm (see top plot of figure 15 , dotted line).

It is also interesting to consider the magnetic energies of poloidal harmonics coupled to the $(2,1)$ mode, which is another signature of toroidal curvature. A larger coupling to adjacent poloidal harmonics would reinforce curvature effects. In the bottom plot of figure 15 , we show the magnetic energies of the $(1,1)$ and $(3,1)$ modes normalized to that of the $(2,1)$ mode, in the linear phase, as a function of Prm. Note that the $(1,1)$ mode is non-resonant since $q>1$. In the low viscosity regime, toroidal mode coupling evolves with an increased coupling to the $m=1$ non-resonant mode, and a lower coupling to $m=3$. It is difficult to extract, from these two opposite variations, the overall effect of the modification of magnetic structure due to viscosity, but it is remarkable that such a modification does appear given the low Prm. In the viscous regime, the mode coupling to adjacent poloidal harmonics decreases for both $m=1$ and $m=3$, thus 


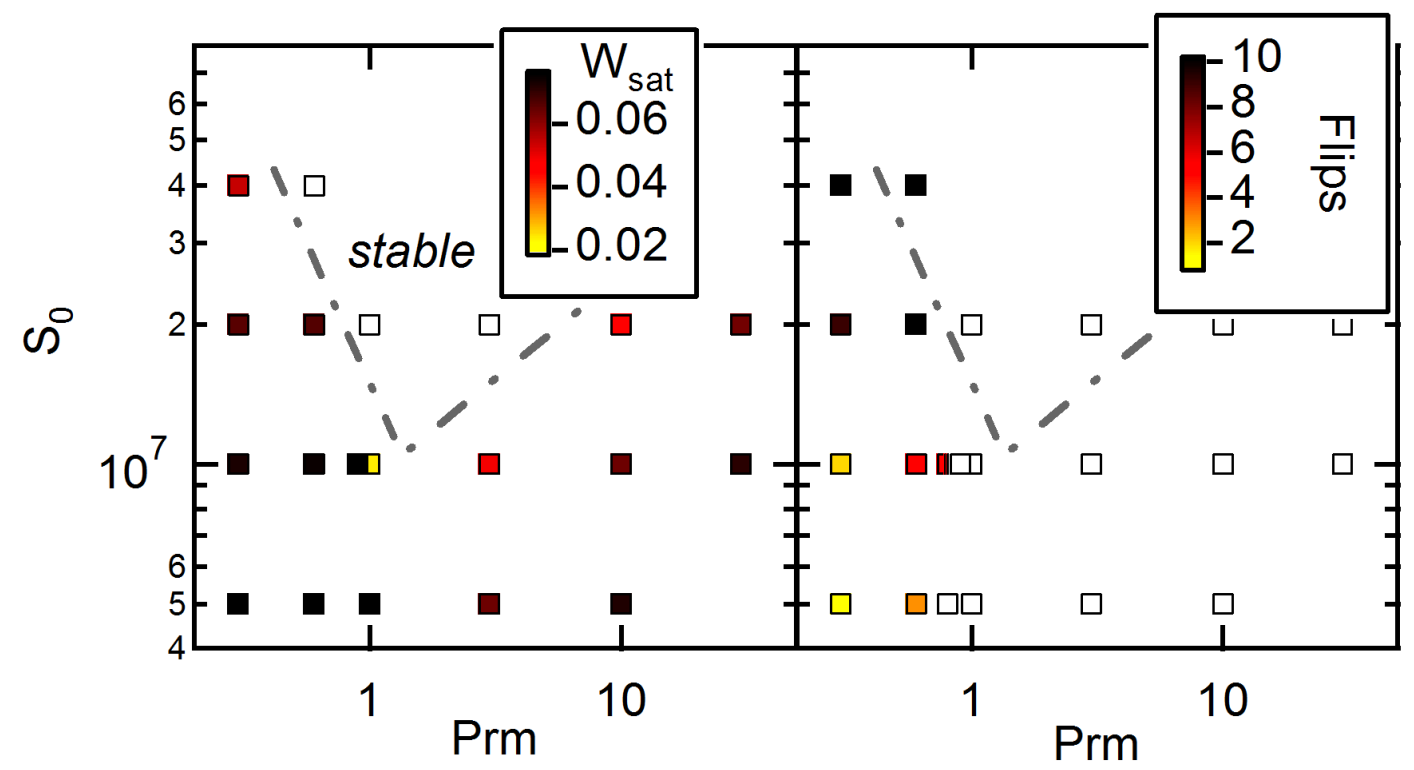

Figure 16. Nonlinear regimes in (Prm, $\left.S_{0}\right)$ plane: island saturation (left), and number of flips (scale limited to 10) (right). The MHD stable region is separated by a dotted line for a better visibility.

indicating a lower effect of curvature in the dynamics of the $(2,1)$ mode. This variation leads to a conclusion that is consistent with that obtained from the change in the perturbed pressure, although the relation between the two approaches could be rather complex. From the elements shown here, we obtain that the broadening of the velocity perturbation, which is the key parameter for the modification of curvature physics, is directly related to Ohm's law, as confirmed by the similar variation of $\Delta_{H W}(\delta V)$ and $\delta_{\eta \nu}$. The magnetic energy coupled to poloidal side-bands characterizes the structure of the perturbed magnetic field, and therefore relates to the same Ohm's law. The correlation between a local approach through the width of the perturbed velocity at the resonant surface, and a global approach via the coupling to adjacent modes, tells us that a stronger magnetic coupling is associated to a narrower velocity perturbation at the resonance.

In the nonlinear regime, curvature physics tend to vanish when the island overcomes a size given by $W_{D}[21,22]$. This is represented in a Rutherford-like framework by:

$$
d W / d \tau_{\eta}=a \Delta^{\prime}(W)-6.35 D_{R} / \sqrt{W^{2}+W_{D}^{2}}
$$

with $\tau_{\eta}=1.22 \mathrm{t} / \mathrm{S}$. The characteristic width $W_{D}$ has been determined from a pure diffusive model for the pressure dynamics by considering only the temperature perturbation [27]. Both the weakening of curvature physics in the linear regime and the increase of the saturation size with Prm indicate that convection should be part of the threshold $W_{D}$. Another indication that claims for a role of curvature physics in 


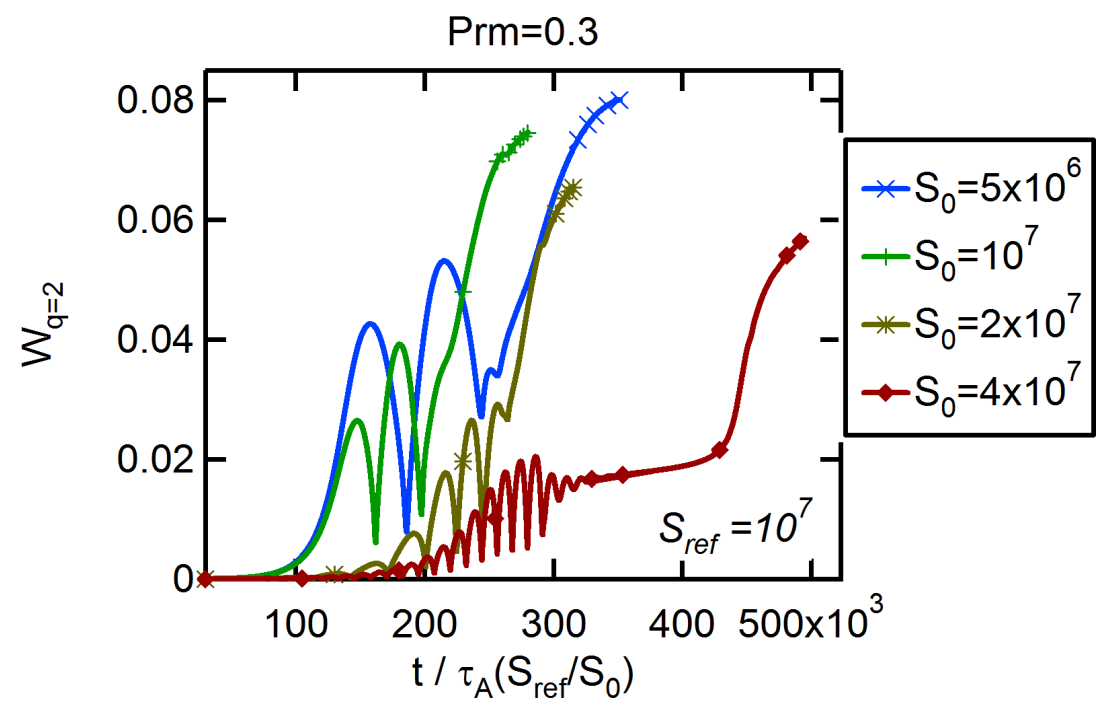

Figure 17. Dynamics of $(2,1)$ island at increasing Lundquist number $S_{0}$ for $\operatorname{Prm}=0.3$ (with $S_{\text {ref }}=10^{7}$ ).

the increase of saturation with Prm is that this variation is not observed in cylindrical models [9]. The progressive increase of the saturation size with Prm is therefore the consequence of a weaker impact of curvature stabilization, due to the broadening of the pressure perturbation through its density contribution that is sensitive to convection. For the understanding of nonlinear MHD simulations, the transport width $W_{D}$ can in fact be evaluated more properly as being proportional to the half width of the pressure perturbation in the linear phase.

The map of the island saturation and nonlinear behaviour in the ( $S_{0}$, Prm) plane is shown in figure 16. The flip instability is observed at low Prm, in particular when the stability threshold is approached. This translates into an increase of the number of flips close to to the stable region. They occur also in the linear growth phase at higher Lundquist numbers (figure 17), and even in the stable domain (Prm=0.6, $S_{0}=4 \times 10^{7}$ ), the island decay from the initial perturbation shows spontaneous flips. This means that when dissipation is low, small deviations from pure linear physics are able to trigger a flip of the island phase. This condition of marginal instability was also observed in the reduced MHD model [9]. Far from the stable domain, even at low Prm, the number of flips decreases, and only one occurrence is observed at Prm=0.1 and $S_{0}=5 \times 10^{6}$.

\section{Conclusion}

We show in this work the existence of two linear and nonlinear regimes for tearing modes, controlled by plasma viscosity. The low viscosity regime is characterized by a growth rate that strongly decreases with the magnetic Prandtl number Prm, as expected from linear theory, and a nonlinear dynamics where the phases of the poloidal harmonics coupled 
by toroidal geometry change several times before saturation is reached. It first occurs on poloidal sidebands of the dominant instability on $q=2$, and seems to propagate to the $(2,1)$ mode so as to minimize the potential energy of the plasma. This adjustment of the island phase, known as the flip instability in situations where reconnection is imposed by an external system such as magnetic coils or localized current drive, occurs spontaneously in this low viscosity regime, and becomes all the more frequent as the linear stability threshold is approached. Strong zonal flow shear is driven in the island region, but it does not appear to impact the flip instability, the exit from the flip cycle nor the saturated size. The transition to the viscous regime occurs when curvature physics starts being affected by viscosity. This takes place when convection takes a sufficient part in the pressure perturbation so that the broadening of the electrostatic potential by viscosity also broadens the perturbed pressure. The linear stability of the tearing mode is then a balance between the stabilizing influence of Prm in the absence of curvature and a lower curvature stabilization at increasing Prm. In the nonlinear regime, the flip instability is absent, and the saturation increases with Prm as a consequence, again, of a weaker curvature stabilization. Previous works performed in slab or cylindrical geometry could not capture this viscous regime, which only exists because toroidal curvature stabilization is modified by viscosity. But they could find the flip instability when considering tearing modes sufficiently close to marginal stability.

This effect of plasma viscosity on tearing mode dynamics could manifest itself in experiments, and affect projections to future tokamaks. Indeed, the dimensionless scaling of the magnetic Prandtl number with respect to $\beta \equiv \mu_{0} p / B^{2}, \rho^{*} \equiv \rho_{s} / a$ and $\nu^{*}=\nu_{i} a / v_{T e}$ differs whether it is controlled by collisional or turbulent processes (here $\rho_{s}$ is the sound Larmor radius, $a$ is the minor radius of the torus, $\nu_{i}$ is the ion collision

frequency and $v_{T e}=\sqrt{2 T_{e} / m_{i}}$ is the electron thermal velocity). In the collisional case, $\operatorname{Prm} \propto \beta$ [28] and remains of order unity or below in standard tokamak discharges. In the turbulent case, $\nu \sim \chi_{\perp}$ and $\operatorname{Prm} \propto \beta / \nu^{*}$, would lead to very large Prm in performant weakly collisional plasmas like in ITER.

\section{Acknowledgments}

We acknowledge the suggestion of one of the referees to deeper investigate the role of flows in the low viscosity regime. This work has been carried out within the framework of the French Research Federation for Fusion Studies. We benefited from HPC resources from GENCI (project 056348) and from Aix-Marseille Université project Equip@Meso (ANR-10-EQPX-29-01) of the program "Investissements d'Avenir" supervised by the Agence Nationale pour la Recherche. This work is part of the project AMICI (ANR14-CE32-0004-01) funded by the Agence Nationale pour la Recherche.

\section{References}

[1] R. Carrera, R. D. Hazeltine, and M. Kotschenreuther. Island bootstrap current modification of the nonlinear dynamics of the tearing mode. Physics of Fluids, 29(4):899-902, 1986. 
[2] R. Fitzpatrick. Interaction of tearing modes with external structures in cylindrical geometry. Nuclear Fusion, 33(7):1049, 1993.

[3] A. I. Smolyakov, A. Hirose, E. Lazzaro, G. B. Re, and J. D. Callen. Rotating nonlinear magnetic islands in a tokamak plasma. Physics of Plasmas, 2(5):1581-1598, 1995.

[4] Y. Ishii, M. Azumi, and A.I. Smolyakov. Nonlinear evolution and deformation of driven magnetic islands in rotating plasmas. Nuclear Fusion, 47(8):1024, 2007.

[5] Qiming Hu, Q. Yu, Bo Rao, Yonghua Ding, Xiwei Hu, Ge Zhuang, and the J-TEXT Team. Effect of externally applied resonant magnetic perturbations on resistive tearing modes. Nuclear Fusion, 52(8):083011, 2012.

[6] Francesco Porcelli. Viscous resistive magnetic reconnection. Physics of Fluids, 30(6):1734-1742, 1987.

[7] D. Grasso, R. J. Hastie, F. Porcelli, and C. Tebaldi. Critical delta' for stability of viscoresistive tearing modes. Physics of Plasmas, 15(7):072113, 2008.

[8] F. Militello, D. Borgogno, D. Grasso, C. Marchetto, and M. Ottaviani. Asymmetric tearing mode in the presence of viscosity. Physics of Plasmas (1994-present), 18(11):112108, 2011.

[9] R. Coelho. Nonlinear growth of marginally unstable tearing modes. Physics of Plasmas (1994present), 14(5):052302, 2007.

[10] A Poyé, A I Smolyakov, O Agullo, S Benkadda, and X Garbet. Saturation of magnetic islands in equilibria with a finite current gradient. Part II: numerical simulations. Plasma Physics and Controlled Fusion, 56(12):125005, 2014.

[11] Hinrich Lütjens and Jean-François Luciani. XTOR-2F: A fully implicit Newton-Krylov solver applied to nonlinear 3D extended MHD in tokamaks. Journal of Computational Physics, 229(21):8130 - 8143, 2010.

[12] D.A. Monticello, R.B. White, and M.N. Rosenbluth. Nonlinear drift tearing modes. Plasma Physics and Controlled Nuclear Fusion Research, 1(IAEA-CN-37/K-3):605, 1978.

[13] E. Lazzaro and M. F. F. Nave. Feedback control of rotating resistive modes. Physics of Fluids (1958-1988), 31(6):1623-1629, 1988.

[14] D. Borgogno, L. Comisso, D. Grasso, and E. Lazzaro. Nonlinear response of magnetic islands to localized electron cyclotron current injection. Physics of Plasmas (1994-present), 21(6):060704, 2014.

[15] H. Lütjens, A. Bondeson, and O. Sauter. The CHEASE code for toroidal MHD equilibria. Computer Physics Communications, 97(3):219-260, 1996.

[16] R. J. La Haye and R. J. Buttery. The stabilizing effect of flow shear on $\mathrm{m} / \mathrm{n}=3 / 2$ magnetic island width in DIII-D. Physics of Plasmas, 16(2):022107, 2009.

[17] R. J. La Haye, D. P. Brennan, R. J. Buttery, and S. P. Gerhardt. Islands in the stream: The effect of plasma flow on tearing stability. Physics of Plasmas, 17(5):056110, 2010.

[18] Giorgio Einaudi and Franco Rubini. Resistive instabilities in a flowing plasma. II. effects of viscosity. Physics of Fluids B: Plasma Physics, 1(11):2224-2228, 1989.

[19] D. Chandra, A. Thyagaraja, A. Sen, C.J. Ham, T.C. Hender, R.J. Hastie, J.W. Connor, P. Kaw, and J. Mendonca. Modelling and analytic studies of sheared flow effects on tearing modes. Nuclear Fusion, 55(5):053016, 2015.

[20] P. Maget, H. Lütjens, M. Brix, P. Buratti, R.J. Buttery, R. Coelho, F. Halpern, N. Hawkes, I. Jenkins, C. Challis, C. Giroud, X. Litaudon, J. Mailloux, N. Mellet, D. Meshcheriakov, and JET EFDA Contributors. Modelling of $(2,1)$ NTM dynamics with flow in JET advanced scenarios. Nuclear Fusion, 51(8):083046, 2011.

[21] Hinrich Lütjens, Jean-François Luciani, and Xavier Garbet. Nonlinear three-dimensional MHD simulations of tearing modes in tokamak plasmas. Plasma Physics and Controlled Fusion, 43(12A):A339-A348, 2001.

[22] M. Kotschenreuther, R. D. Hazeltine, and P. J. Morrison. Nonlinear dynamics of magnetic islands with curvature and pressure. Physics of Fluids, 28(1):294-302, 1985.

[23] Hinrich Lütjens, Jean-François Luciani, and Xavier Garbet. Curvature effects on the dynamics of 
tearing modes in tokamaks. Physics of Plasmas, 8(10):4267-4270, 2001.

[24] D Edery and A Samain. Toroidal coupling and frequency spectrum of tearing modes. Plasma Physics and Controlled Fusion, 32(2):93, 1990.

[25] A. Ali, Jiquan Li, and Y. Kishimoto. On the abrupt growth dynamics of nonlinear resistive tearing mode and the viscosity effects. Physics of Plasmas, 21(5):052312, 2014.

[26] A. H. Glasser, J. M. Greene, and J. L. Johnson. Resistive instabilities in general toroidal plasma configurations. Physics of Fluids, 18(7):875-888, 1975.

[27] Richard Fitzpatrick. Helical temperature perturbations associated with tearing modes in tokamak plasmas. Physics of Plasmas, 2(3):825-838, 1995.

[28] S.I. Braginskii. Transport processes in plasma. In M.A. Leontovich, editor, Review of Plasma Physics, volume 1 of Review of Plasma Physics, pages 201-311. Consultants Bureau, New York, U.S.A., 1965. 